\section{TRUE COLOUR SEM IMAGING FOR PHASE RECOGNITION AMD X-RAY MICROANALYSIS}

Peter J.Statham

Oxford Instruments Microanalysis Group

Secondary (SE) and backscattered electron (BSE) signals in the SEM provide high resolution monochrome images. BSE signal strength is modulated by mean atomic number and 'false" colour can be introduced to enhance material contrast. Colour can also be introduced using multiple SE detectors, each with a different sensitivity to topographic and compositional information: by controlling signal mixtures and colours, the operator effectively has access to a powerful "studio" to generate aesthetically pleasing colour images. ${ }^{1}$ In both these examples, the correspondence between local elemental content and colour is entirely arbitrary and under subjective control of the operator. Elemental x-ray maps can be acquired and combinations colour coded to reveal phase distributions. ${ }^{2}$ For large numbers of maps and images, chemometric techniques such as PCA may be used to discover common relationships and assist the process of colour coding. ${ }^{3}$ Images derived from x-ray maps are usually low resolution and the analyst has to decide which elements to include and do a fair amount of data manipulation before any conclusions can be drawn. Furthermore, local topography effects perturb any multivariate statistical analysis. This paper presents a novel imaging technique which addresses these limitations.

In cathodoluminescence (CL), the sample emits visible light, and images can be coded to reflect the "true colour", as would be seen in an optical microscope. ${ }^{4}$ By analogy, we can use the emitted $x$-ray signal to generate a true colour image. Figure 1 shows the normal human visual response to light with wavelengths from about 400 to $700 \mathrm{~nm}$. To offset the visual response into the $\mathrm{x}$-ray region, an energy dispersive $\mathrm{x}$-ray detector and analysis system (i.e., Link ISIS) is used to assign colour to photons based on their effective wavelengths using a rainbow colour scale. For example, with a 300 $\mathrm{eV}$ x-ray assigned red and a $5 \mathrm{keV}$ photon assigned blue, the offset visual response appears as in the lower graph in Figure 1. While the electron beam is resting on a point on the sample, as each photon is detected, its energy is measured and converted to a colour $(r, g, b)$ with the condition $r+g+b=1$ and the results added to three colour accumulators which maintain totals $R, G, B$. At the end of the acquisition period, $R, G, B$ effectively represents the colour sensation to the emitted $x$-ray spectrum which can be reproduced by display on a colour monitor. This process is repeated for all the pixels in a rectangular grid over the field of view to build up a complete full colour image in approximately 1 minute; the acquisition can be extended for further frames to improve counting statistics. The resultant colour $\mathrm{x}$-ray image effectively provides a low bandwidth chrominance signal which is combined with a high resolution monochrome electron image previously acquired from the same field of view using the same beam control hardware. As in conventional broadcast television, the combination is designed to maintain the same luminance as the electron image and thus retain high resolution image detail and topographic appearance. ${ }^{5,6}$

Figure 2 still exhibits the topography and fine detail of the SE image. After the true $x$-ray colour is added, the tungsten-rich balls appear green, the titaniumrich balls appear blue and the carbon cement substrate is tinged red. If the red, green and blue components for each pixel are plotted on a ternary diagram the resulting scatter plot reveals clusters corresponding to the regions of similar colour (Figure 3). In a multiphase sample, the numbers of pixels in each cluster can be used to calculate area $\%$ for each phase.

The multidimensional information corresponding to individual spectra at every pixel point is effectively compressed into a single true colour image and although separation of phases into different colours is not always dramatic, phase recognition is considerably easier and more intuitive than multivariate statistical analysis. Provided the same energy response band is used, the colour will be reproduced on any microscope under similar conditions so compounds will

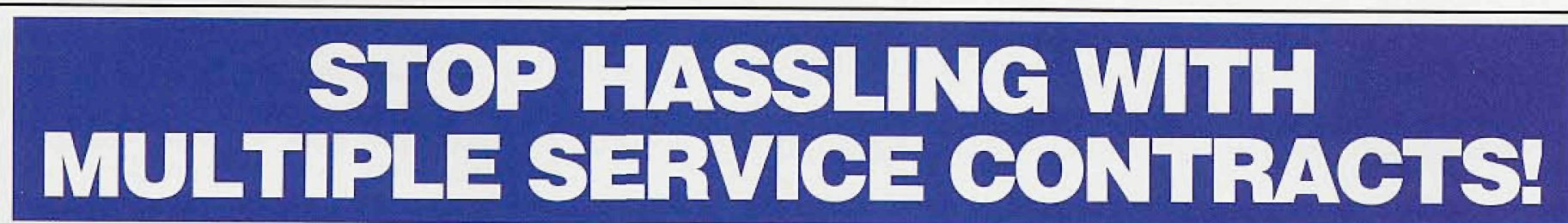

START by putting all of your instruments under one contract with MAS

(regardless of make or model). Our expert EM SERVICE GROUP has the knowledge and skill to keep your instrumentation working at its best.

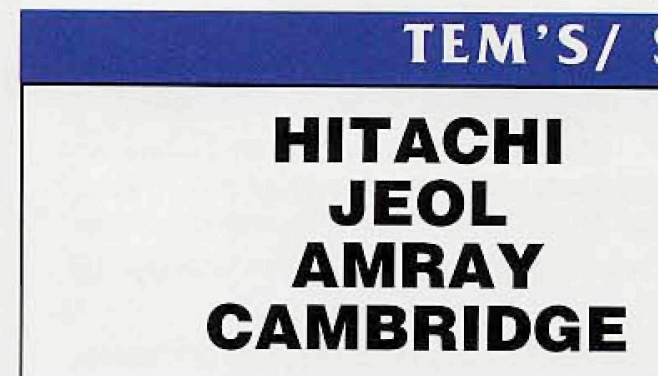

We service all brands under one contract.
TOPCON ISI ZEISS PHILIPS

\section{PREP EQUIPMENT}

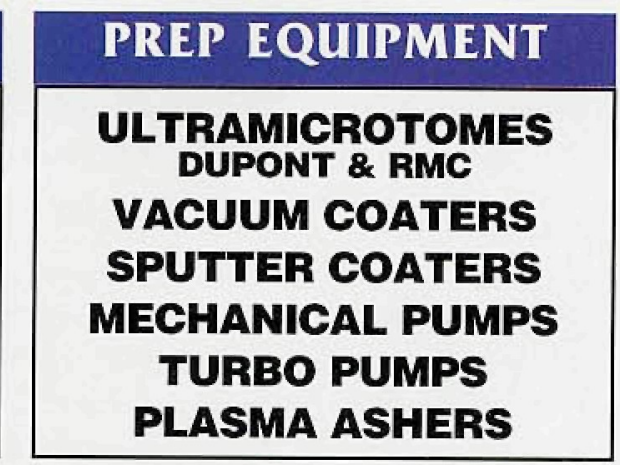

WE SERVICE COMPUTER CONTROLLERS BEAM BLANKERS WATER CHILLERS
Contracts and On-Demand Emergency Service at Reasonable Rates from Factory Trained Specialists. 1-800-421-8451

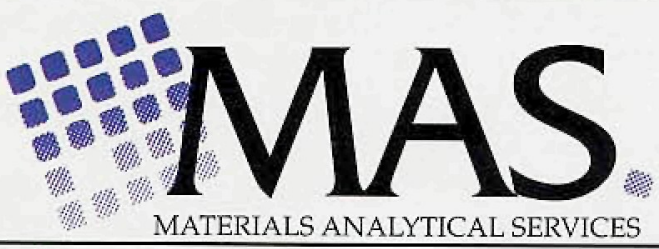

MATERIALS ANALYTICAL SERVICES
3597 Parkway Lane - Suite 250 • Norcross, Georgia 30092 • 770-448-3200 • FAX 770-368-8256 or 616 Hutton Street • Suite 101 • Raleigh, North Carolina 27606 • 919-829-7041 • FAX 919-829-5518 ADVANCED ANALYTICAL PRODUCTS AND SERVICES 


\section{True Color SEM Imaging....Continued from Page 8}

appear in memorable colours which can be learned for $\mathrm{CL}$ microscopy Unlike pseudo-colour encoding of BSE images or signal mixing techniques, the colour is independent of beam current, insensitive to local geometry and no user intervention is required to calibrate thresholds. Unlike conventional $x$-ray mapping techniques, there is no need to choose specific elements and set appropriate windows. The augmented image is intuitive and easy to interpret yet contains a considerable amount of encoded information equivalent to a multitude of $x$-ray images. A single image can be acquired in about one minute with sufficient statistics to give an informative "first look" at a sample. Faced with a complex analytical problem, the analyst can use this true colour $\mathrm{x}$-ray imaging technique to give a useful cue for positioning the probe for subsequent microanalysis, obtain area $\%$ of phases or simply to compress topographical and compositional information into a single image for archiving.

\section{D. Scharf, U.S. Patent 5212383, May 18, 1993}

2. P.J.Statham and M.Jones, Scanning, 3(1980) 168-171

3. R. Browning, Surface and Interface Analysis, 20(1993)495-502

4. G.V.Saparin and S.K.Obyden, Scanning, 10(1988)87-106

5. P.Statham, U.S. Patent 5357110, Oct 18,1994

6. P.Statham, Microchimica Acta, in press, Springer Verlag (1996)

7. I am grateful to my colleagues, particularly Neville Cox for software implemetation and Judith Root, Mohinder Dosanjh and Pierre Rolland for experimental work.
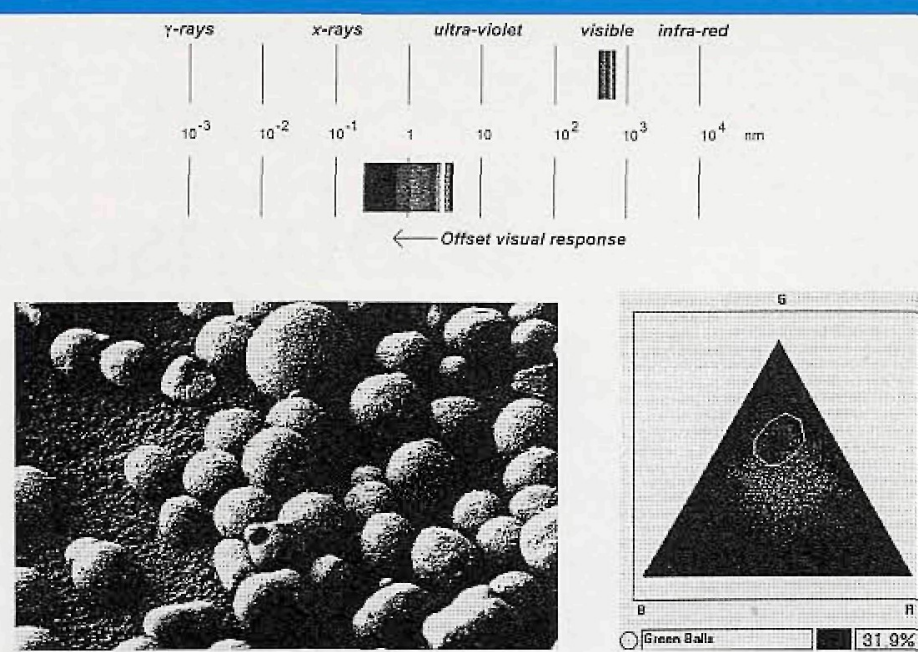

Figure 1: Electromagnetic spectrum showing region where human visual sensors normally operate to give the sensation of colour. Offsetting this visual response allows us to see a true colour $x$-ray image.

Figure 2: Monochrome reproduction of colour augmented image. $20 \mathrm{kV}$. field width 1000 $\mu \mathrm{m}$, acquire time $3 \mathrm{~min}$.

Figure 3: Ternary scatterplot showing colour distribution for fig.2. Outlined region gives total area for $W$ phase.

Reprinted from the Proceedings of Microscopy \& Microanalysis '96, the conference held by the Microscopy of America
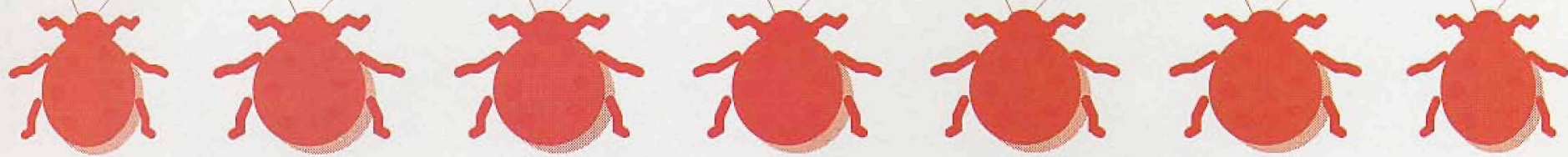

\section{When Solutions Count... The Result $=\mu$ View}

\section{Microscope Video Image Software!}

Spectra-Tech announces the introduction of $\mu$ View (Micro View), a powerful and versatile new Video Imaging Software for Visible and Infrared Microscopes. This. Windows-compatible package greatly enhances sample viewing, image capture, annotation and documentation.

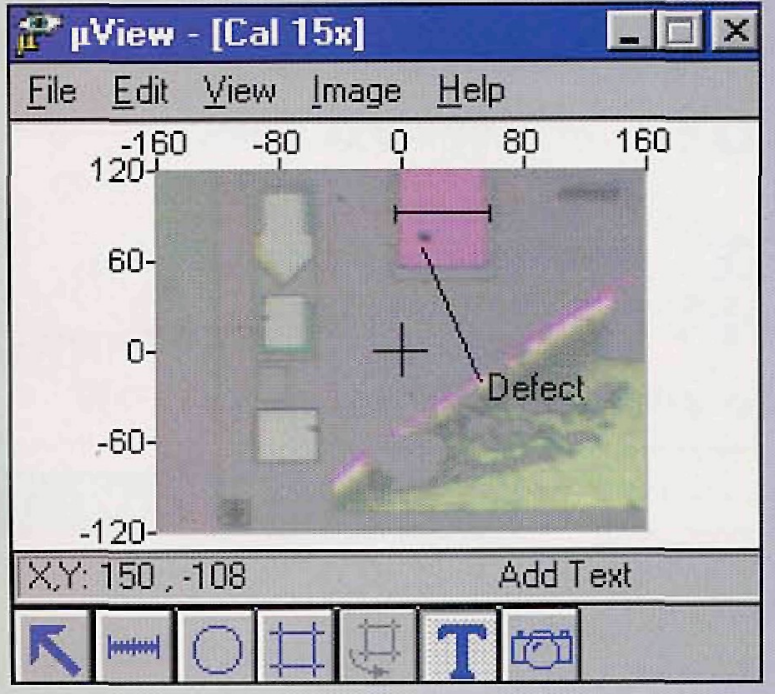

SPECTRATECHM

\section{Features:}

- Live sample image appears in a video pane in the Windows application

- Annotation, printing and saving measurements of sample dimensions

- Intuitive and easy to use

- Produces image files that contain true colors

- A scale surrounds the video image \& allows the viewer to determine the size of sample

- A cross hair displayed over the sample image indicates the center of the field of view

- Variety of useful tools

\section{$\mu$ View}

Spectra-Tech Inc., 2 Research Drive, Shelton, CT 06484-0869 Phone: 1-800-243-9186, CT 203-926-8998, Fax: 203-926-8909 

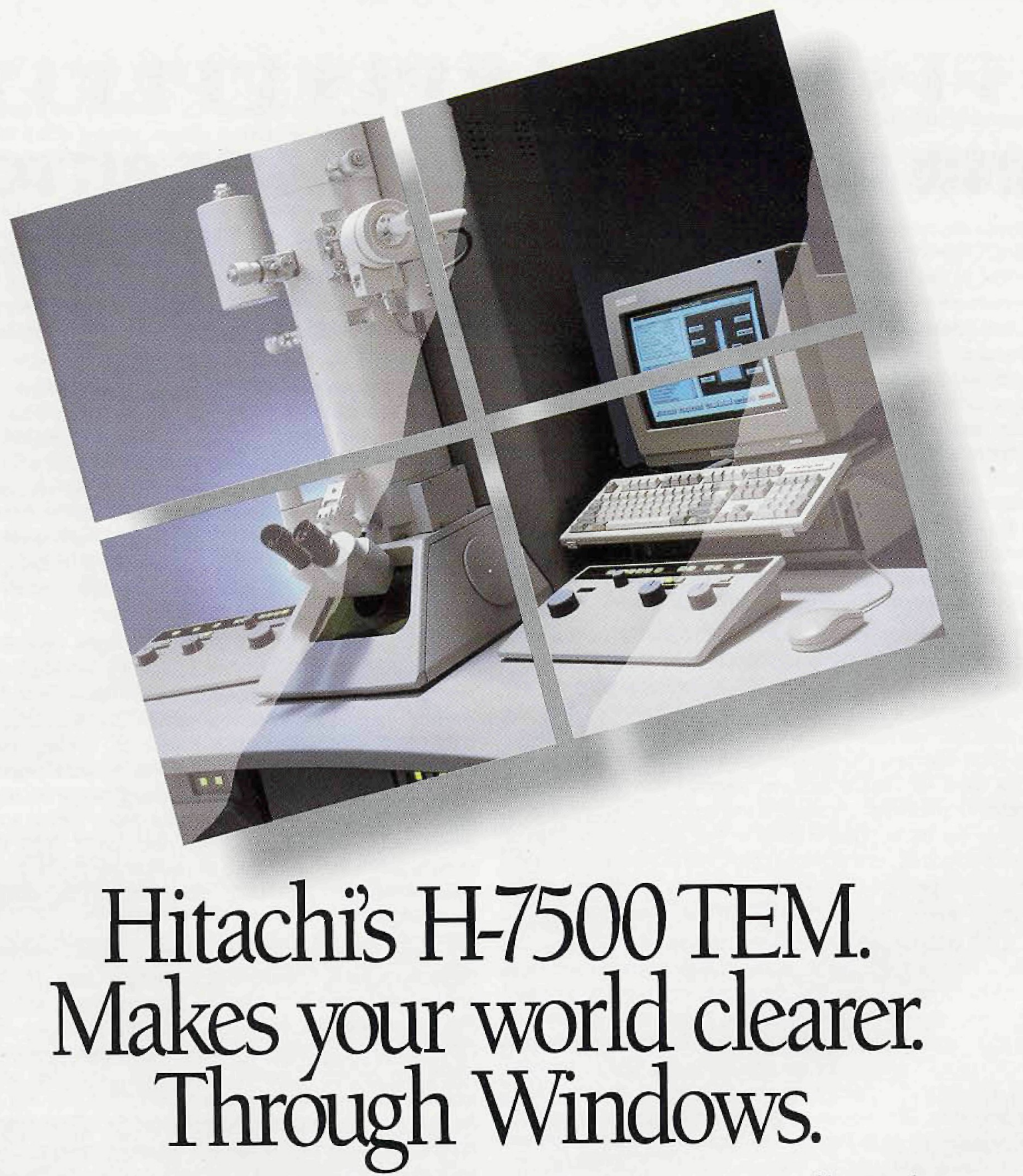

High performance. Sophisticated functions. Extended features. In biological research TEMs, all that can add up to operational complexity.

Not with our H-7500 TEM. It lets you acquire quality images, perform detailed analyses, using a friendly Window ${ }^{\mathrm{TM}}$ interface. Replacing perplexity with productivity.

But beyond simple control, you can count on truly clean images. With superior resolution enhanced by a column design ensuring optimized gun brightness at any accelerating voltage. With a special high-contrast mode made possible by a computercontrolled objective lens that allows the use of smaller apertures - critical to contrast - without limiting field of view.

Our small-footprint H-7500 also simplifies selection of fields of interest and beam positions by linking control of its patented-design Hiper-Stage to magnification, so specimen traverse speed on the screen is the same at any magnification. Other efficiency-boosting features range from automatically controlled and optimized beam current to half-frame photography for combined survey/zoom and stereo-pair film records.

Call, visit our Web site, or E-mail us for details. Better yet, arrange to run some samples at a personal demonstration. That way you'll get the whole picture. And a clear one. Easily.

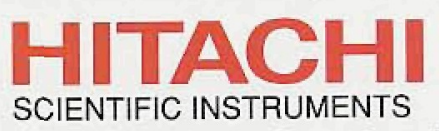

Nissei Sangyo America, Ltd.

755 Ravendale Drive

Mountain View, CA 94043

(800) 227-8877

E-mail: sidsales@nissei.com wwwnissei.com

25 West Watkins Mill Road Gaithersburg, MD 20878 (800) 638-4087 